\title{
POLÍTICAS PÚBLICAS... PROCESO COMPLEJO, REFLEJO DE LAS RELACIONES DE PODER DEL SISTEMA POLIIIICO*
}

KATHERINETABARES

\author{
RESUMEN
}

\begin{abstract}
La política pública es un conjunto de acciones continuas adelantadas por una autoridad pública, orientadas al manejo de una situación socialmente problemática que ha logrado ubicarse en la agenda pública (condición principal para ser objeto de una política) por presión de ciertos sectores y grupos de interés que le han introducido entre las respuestas de acción de las autoridades y administración de turno. A partir de esta definición en el presente artículo se realiza una reflexión frente a la complejidad de las políticas públicas, precisando como éstas surgen y tienen lugar en medio de un juego de relaciones de poder que se imponen en el proceso de la toma de decisiones, fundamental en la definición de la política.
\end{abstract}

Palabras claves: Política, Proceso, Decisiones.

\begin{abstract}
Public policy is a series of continual actions carried out by a public authority, oriented toward the management of a socially problematic situation that has arrived at the public agenda (basic requirement for being the object of a policy) for the pressure of certain sectors and interest groups that have introduced it among action responses to the authorities and the acting administration. From this definition in this article one reflects upon the complexity of public policies, clarifying how these come about and play a role in the relations of power that impose themselves in the process of decision making, fundamental in a definition of policy.
\end{abstract}

Key words: Policy, Process, Decisions.

* Ensayo elaborado para el seminario de formulación de Políticas Públicas con el profesor Víctor Mario Estrada Ospina. Universidad del Valle. 

discusión y reflexión de la tarea compleja y por ello nada fácil del análisis del proceso de la política pública, pues ésta por su naturaleza y desarrollo en el escenario creado por el sistema político (particular de un sociedad en un momento histórico) hace que intervengan diversos actores, con intereses particulares y ocupantes de una posición de poder. Es por ello que un análisis del proceso de la política pública implica tener en cuenta a estos actores, con sus posiciones, relaciones de poder, contexto económico, político, social. Es así como el analista de políticas públicas se ubica en un sistema político, entendiéndola como un conjunto de acciones continuas adelantadas por una autoridad pública, orientadas al manejo de una situación socialmente problemática que ha logrado ubicarse en la agenda pública (condición principal para ser objeto de una política) por presión de ciertos sectores y grupos de interés que le han introducido entre las respuestas de acción de las autoridades y administración de turno.

De acuerdo con lo anterior es importante precisar que los problemas que dan lugar a la política son construcciones sociales que reflejan construcciones específicas de la realidad, es así como una definición de una política pública evidencia un marco conceptual de ver la realidad, una forma de operar de una autoridad gubernamental, de abordar y tratar una situación; a partir de esto se denota como la subjetividad y las tramas de relación y de poder juegan un papel significativo en el escenario de las políticas.

Con todo esto, en las reflexiones realizadas en el seminario y las lecturas que han soportado las discusiones se ha evidenciado como una forma apropiada (aunque no del todo) para el análisis del proceso de la política pública es dividir el proceso en fases: formulación, diseño de la política, implementación y evaluación, que de manera analítica pueden hacer que se comprendan los elementos que hacen posible la política y el desarrollo de la misma, aunque estas no se dan de manera lineal ya que la circularidad es una de las características del proceso. Finalmente una constante del análisis es enmarcarse en el sistema de actores involucrados, el proceso y la actividad.

Hecha esta pequeña introducción me ocupare en el presente ensayo de una fase del proceso de la política, la toma de decisiones, que presenta las tensiones señaladas por el objeto de las políticas y teniendo presente que se desarrolla durante todo el proceso. El escrito se desarrollara con el siguiente esquema, precisión del campo social como contexto de la política, traducción de la acción gubernamental a través de la política y finalmente tensiones de la toma de decisiones.

Me parece pertinente señalar antes de hacer alusión a la etapa concretamente, como siendo el contexto y escenario de la política el campo social, el analista ha de tenerlo presente en su análisis pues éste si se entiende como el lugar donde se configura una dinámica relación y soporte de las diferentes posiciones y roles de los sujetos, precisarlo permite identificar la particularidad de los sistemas políticos donde se desarrolla la tarea del diseño y ejecución de la política.

Siendo el sistema político la evidencia y desarrollo de un sistema democrático donde circulan los derechos y las demandas del colectivo, el análisis de él para el analista será una radiografía de las estrategias discursivas e ideológicas de los actores del campo social, en una estructura económica, política, social y cultural inscritos en un juego donde su única finalidad es la materialización de 
sus intereses, donde la política pública aparece como camino y vehículo para llegar a su meta.

En este contexto la acción del Estado se traduce en los sistemas políticos en una gobernabilidad particular, es decir el programa de acción y las actividades que lleva acabo en un periodo de tiempo un funcionario de turno, que toma decisiones, frente a las posibles soluciones de las consideradas situaciones socialmente problemáticas. De éste modo el analista tendrá en cuenta que dichas decisiones están mediadas por los actores involucrados en el proceso (autoridades publicas y gubernamentales) (Rot D, y Andre N. 2002), quienes tienen intereses y recursos materiales e ideológicos mediados por sus mapas cognoscitivos de la realidad y que por ello habrá que mirarlos en el campo social en su organización social, su influencia y capacidad de movilización para desarrollar sus estrategias y realización de objetivos.

A partir de lo anterior me introduzco en la discusión del presente ensayo, cómo entender el proceso de toma de decisiones para el analista de políticas en el campo social.

Las decisiones son vistas como el resultado de una confluencia de cuatro corrientes: las personas, problemas, las soluciones y las oportunidades de elección. Todas están inmiscuidas en tramas de relación que terminan articulándose o imponiéndose entre sí de acuerdo con las estrategias de poder del gobierno, población afectada o grupos de presión e interés y actores políticos presentes en el proceso.

¿Por qué se habla de estrategias de poder? Porque aunque quien tiene el control de la agenda son las autoridades, estas deben permitir la participación de otros actores de la sociedad civil, aunque participantes en la medida que cuentan con la capacidad de movilización y ejercer presión para vehiculizar sus demandas, deben elaborar una propuesta clara en cuanto los factores, variables incidentes y estrategias de acción modificantes de la situación problemática. Pero a pesar de esto la participación en la formación de la agenda de las instituciones suele inclinarse en favor de algunos grupos y cuestiones con exclusión de otras agrupaciones y asuntos, por las posiciones de poder que pueden ponerse en juego o a las que se quiera acceder.

Elegido un problema, se pasa a la definición del problema la cual esta mediada por los mapas cognoscitivos de los participantes del proceso y del mismo modo por la visualización de las soluciones del problema de acuerdo con los recursos y organización burocrática. Aquí empezamos a entrarnos en otra de las tensiones del proceso de la política, concreta de la etapa de decisión: "formulación de soluciones y legitimación de la solución".

En la primera etapa se hace énfasis en el planteamiento de soluciones de lo que se considere como competente con las propuestas y alternativas de soluciones planteadas, a las que le subyacen un punto de vista, de comprensión de la realidad y de análisis del problema de los diversos actores que exponen sus argumentos y contra argumentos para hacer imponer su visión.

\section{¿QUIÉNES PARTICIPAN EN ESTE PROCESO?}

Participan los sectores y grupos de interés que por su posición y acción en el campo social permite que su voz sea escuchada y su presión logre calarse en las instancias de poder, de este 
modo este proceso esta mediado por las relaciones de poder, donde en ocasiones los más afectados no logran evidenciar su posición ya que no cuentan con los recursos materiales e ideológicos para ubicarse en la competencia frente a los sectores de poder que del mismo modo ven un beneficio en el planteamiento de la política, así la selección de alternativas de los rumbos de acción de la política estará determinada por el juego de poderes. Como lo dice Rot, "en esta fase interactúan actores de acuerdo a la apertura y permeabilidad del sistema politico".

Es importante mencionar que son dos lo parámetros que están más presentes en el proceso decisional, (Meny I y Thoenig J. 1992) los cuales lo ubican en el funcionamiento del sistema político, son el grado de acuerdo y desacuerdo entre los que participan en la decisión acerca de los objetivos y valores ligados al problema y el grado de certeza en cuanto a qué medios deben utilizarse.

Seleccionadas las alternativas de solución se entra a la etapa de la legitimación la cual esta referida a la forma en que la figura política, que ostenta la autoridad y el poder legal (alcalde, gobernador, funcionario público) en la toma de decisiones, por medio de un acto ritual, presenta ante terceros la autoridad y fuerza real de la decisión en el campo social.

Otros elementos que están presentes en el sistema político a la hora de diseño de las políticas y que están directamente inmiscuidos con las estrategias y juegos de poder son dos cuestiones que analizados reflejan los intereses y las relaciones sociales presentes que tienen peso en una opción a escoger. Los dos puntos son: las concepciones erróneas de las opciones de solución a los problemas de política y los modos incorrectos de elaborar las propuestas alternativas. (Meny I y Thoenig J. 1992).

\section{¿Cómo ba de analizar el analista este momento del proceso de la política?}

Es preciso aclarar primero que se entiende por opciones de politica las hipótesis tentativas acerca de cuáles cursos de acción pueden conducir a resolver los diferentes aspectos de los problemas existentes, considerando en su propuesta de solución las dimensiones políticas, legales, administrativas y económicas de toda politica (Meny I y Thoenig J. 1992).

Teniendo en cuenta esta definición vemos cómo la toma de decisiones siendo un proceso mediado por relaciones de poder, modos de abordar la realidad de acuerdo a particulares intereses, no se escapa de presentar a quienes asumen la responsabilidad de tomar las decisiones, obstáculos frente a la decisión de cual será la dirección y la finalidad de los que serán los objetivos y metas de la política; haciéndose más compleja esta situación al ubicar este proceso en un campo social donde hay diversos actores intervinientes o actores del proceso con intereses que pueden contraponerse lo que puede llevar a creerse que solo hay un modo de manejar la situación y llegar a la situación deseada; y del mismo modo creerse que los problemas definen las soluciones.

Es por esto que el analista debe obtener la información suficiente para tener claro el alcance y complejidad del problema de modo que pueda identificar los soportes de la situación problemática y pueda elaborar un diagnóstico que le sirva como referente, para intervenir o manipular la situación con sus variables más incidentes mediante una estrategia orientada a una posible forma de conducirla. 
Es aquí donde podría ser una buena herramienta lo que se denomina en el tercer enfoque de la toma de decisiones, el mapeo retrospectivo el cual se ubica en el escenario y momento de la situación problemática para un entendimiento más próximo, (Aguilar L, 2002); se puede en él reconocer el statu quo, las variables y factores intervinientes en la situación que se quiere manejar para así, de cierto modo jugar con los posibles cruces o relaciones que se pueden establecer en las realidades concretas para llevar la situación al estado deseado.

Entender y comprender el problema para señalar la conducción más adecuada, no es una tarea fácil y lineal, pues hay que tener en cuenta que las organizaciones responsables cuentan con determinados recursos que si bien para algunos casos son suficientes, para otros es importante de acuerdo a la distribución que se hace de ellos, determinar las alternativas posibles y factibles desde las realidades particulares, (tomar medidas factibles) esto implica tener un método flexible que sea capaz y acepte reformulaciones de las estrategias contando con los recursos disponibles.

Por ello Aguilar, señala que un buen análisis tiene fuerza en la medida en que esclarece las diferencias que se pueden dar entre las varias respuestas diversas a los problemas, de modo que se pueda dilucidar los ajustes entre los pro y contra de los diferentes objetivos, al considerar como se precede un status quo que puede ser modificado con la alteración y el juego de ciertos factores y variables que constituyen la problemática y que pueden traducir posibles afectos.

Es así como el mapa retrospectivo y la manipulación de variables puede plantear estrategias de acción en las que se tiene en cuenta la proporción de beneficios limitados y específicos que dará respuesta la política (pues hay que tener claro que la política no dará solución completa al problema) a partir del entendimiento del contexto en que opera una organización y del conocimiento específico del programa (es importante que se disponga de la información tanto del programa, como la organización y el contexto problemático) considerando las combinaciones posibles y reformulando estrategias

De este modo se hace el análisis de la realidad de la situación problemática considerando además las variables del entorno político, económico, cultural y social presentes en el proceso de la política pública

Finalmente, es importante describir como se cometen ciertos errores que pueden hacer que los efectos de la política no respondan a los objetivos que beneficien al sector afectado o que simplemente dan cuenta de las dinámicas de poder y de la presión de ciertos grupos que puede hacer que: "se definan mal los problemas, se ataquen los síntomas y no las soluciones, se prescriban alternativas desde arriba hacia abajo especificando los objetivos y las acciones para realizarlos" (Meny I y Thoenig J. 1992).

\section{¿Cómo formular las soluciones?}

Aunque esta pregunta pareciera muy técnica y no resuelva los problemas de la formulación de las soluciones; algunos autores hacen referencia a la toma de decisiones a partir de modelos para planificary tomar decisiones, estos de manera muy personal, son un poco rígidos ya que establecen una posición del decisor y una perspectiva de comportamiento ante las respuestas a los problemas, 
que el autor en la misma exposición evidencia como resultan siendo esquemas insuficientes ante una realidad en la que la previsión de variables se hace compleja ya que se permea por conductas y planes de ciertos sectores de poder que introducen situaciones diferentes que un modelo de planificación no pueda manejar y se quede corto para ilustrar todo un proceso de acción de las autoridades publicas.

Pero si bien planteo esta posición también reconozco algunos aportes Rot, importantes para la formulación por parte del analista:

- Claridad entre los fines o metas de la política, precisando objetivos que responden al por qué y cómo de ella; traducidos además en indicadores que darán cuenta de realización o no de la meta.

De este modo se presenta la planificación como una herramienta metodológica útil para integrar las diferentes políticas sectoriales en un tiempo determinado pues ilustra los objetivos que se quieren alcanzar, definición de prioridades, jerarquización de objetivos a corto y mediano plazo.

Del mismo modo con la planificación se intenta disminuir la incertidumbre del futuro, considerando un relativo control sobre los factores de desarrollo. Pero debe considerarse además cierto grado de flexibilidad de modo que acepte planes emergentes cuando la realidad presente coyunturas que se salen del esquema y de los factores valorados en el proceso.

Así Rot, plantea finalmente que la sustancia de las decisiones depende de la naturaleza de los problemas, del contexto institucional, organizativo y sociopolítico en el que se mueven quienes deciden, esto sustenta la posición presentada a lo largo del texto y es como el campo social, con su dinámica particular y juego de poderes determina la dirección y lógicas que caracterizan las decisiones en el escenario de las políticas públicas.

Finalmente, hay que tener en cuenta que a través de las opciones de una política se intentan manejar más no resolver los problemas públicos, relacionando los medios manipulables y los objetivos alcanzables. La precisión y definición de ellos es lo que se convierte en el cuello de botella del proceso de la política pública por la pluralidad de intereses presentes en el proceso.

\section{REFERENCIAS BIBLIOGRÁFICAS}

MAY J. PETER, “Claves para diseñar opciones de políticas”, en: Aguilar Luis Fernando, Problemas, Grupo

Editorial Porrúa, México, 1993.

meny ives, thoenig, Jen Claude, "La decisión pública", en: Las Políticas Públicas, Editorial Ariel, Barcelona, 1992.

ROT, D, ANDRÉ N. Formulación de Soluciones y Decisión.

AMITAI ETZIONI, La exploración combinada: Un tercer enfoque de la toma de decisiones.

ELDER, CHARLES D, y COBB W ROGER, "La formación de la agenda: El caso de la política de los ancianos", en: Aguilar, Luis Fernando, Problemas Públicos y Agenda de Gobierno, Grupo Editorial Porrúa, México, 1993.

Recibido en mayo 10 de 2004

Aprobado en junio 15 de 2004 